\title{
Waveguide-Plasmon Polaritons Enhance Transverse Magneto-Optical Kerr Effect
}

\author{
Lars E. Kreilkamp, ${ }^{1}$ Vladimir I. Belotelov, ${ }^{2,3}$ Jessie Yao Chin, ${ }^{4, *}$ Stefanie Neutzner, ${ }^{4}$ Daniel Dregely, ${ }^{4}$ \\ Thomas Wehlus, ${ }^{5}$ Ilya A. Akimov, ${ }^{1}$ Manfred Bayer, ${ }^{1}$ Bernd Stritzker, ${ }^{5}$ and Harald Giessen ${ }^{4}$ \\ ${ }^{1}$ Experimentelle Physik 2, Technische Universität Dortmund, D-44221 Dortmund, Germany \\ ${ }^{2}$ Lomonosov Moscow State University, Faculty of Physics, 119991 Moscow, Russia \\ ${ }^{3}$ Russian Quantum Centre, 143025 Skolkovo, Moscow Region, Russia \\ ${ }^{4} 4$ th Physics Institute and Research Center SCOPE, University of Stuttgart, 70550 Stuttgart, Germany \\ ${ }^{5}$ Institute of Physics, University of Augsburg, 86135 Augsburg, Germany \\ (Received 13 March 2013; revised manuscript received 20 October 2013; published 25 November 2013)
}

\begin{abstract}
Magneto-optical effects in ferrimagnetic or ferromagnetic materials are usually too weak for potential applications. The transverse magneto-optical Kerr effect (TMOKE) in ferromagnetic films is typically on the order of $0.1 \%$. Here, we demonstrate experimentally the enhancement of TMOKE due to the interaction of particle plasmons in gold nanowires with a photonic waveguide consisting of magnetooptical material, where hybrid waveguide-plasmon polaritons are excited. We achieve a large TMOKE that modulates the transmitted light intensity by $1.5 \%$, accompanied by high transparency of the system. Our concept may lead to novel devices of miniaturized photonic circuits and switches, which are controllable by an external magnetic field.
\end{abstract}

DOI: 10.1103/PhysRevX.3.041019

Subject Areas: Optics, Photonics, Plasmonics

\section{INTRODUCTION}

The optical far-field property of a magnetic medium can be modulated by its magnetization M. Specifically, under oblique incidence, the amount of light being transmitted and reflected by the medium varies when the transversal magnetization is flipped [1,2]. This effect is termed the transverse magneto-optical Kerr effect (TMOKE). The TMOKE holds application potential for novel nano-optical devices. In particular, optical modulators and switches controlled by an external magnetic field could be realized. However, the TMOKE in ferromagnets such as nickel or cobalt is rather small, and the high losses of these metals hamper their application in optical devices [2]. In the past few years, several concepts were proposed to combine nano-optics and plasmonics with magneto-optical materials [3-31], where nano-optical and plasmonic structures were capable of enhancing or even giving rise to new magneto-optical effects. In particular, a longitudinal magnetic field was shown to be able to modulate the transmission of magneto-plasmonic structures, although the loss of light intensity was over $90 \%$ [21].

Early on, several papers studied the interplay between surface plasmon polaritons (SPPs) and magneto-optics and addressed SPPs propagating along smooth surfaces of ferromagnetic or semiconductor films [32-34]. It was later shown that periodic perforation of ferromagnetic metals provided Fano resonances in the transmission or reflection

*j.chin@ physik.uni-stuttgart.de

Published by the American Physical Society under the terms of the Creative Commons Attribution 3.0 License. Further distribution of this work must maintain attribution to the author(s) and the published article's title, journal citation, and DOI. spectra because of the excitation of SPPs, which led to several times enhancement of the TMOKE [22-25]. To reduce the overall optical losses, bimetallic systems of smooth or perforated noble and ferromagnetic metals were investigated, where the SPPs could propagate for relatively long distances along the air-metal interface, and the TMOKE was enhanced at the ferromagnetic surface [26-31]. By reducing the losses, larger enhancement of the TMOKE was achieved.

Recently, Belotelov and colleagues demonstrated a new concept [3-5], where the ferromagnetic material was substituted by a low-absorptive noble metal and a magnetooptical dielectric layer was introduced. It allowed the longrange SPPs to propagate at the magnetized surface, which produced large enhancement of the TMOKE so that the transmitted light intensity could be modulated by $1.5 \%$ [3]. However, the geometry of perforated metals led to a very small transmittance of $3 \%$ for the SPP-enhanced TMOKE.

In this paper, we experimentally demonstrate a new concept capable of magnetically modulating the transmission of light while simultaneously maintaining a high level of transparency. Our concept incorporates magneto-optical thin films with waveguide-plasmon polaritons [35,36], which stem from the coupling between localized surface plasmons and guided photons. We quantify the TMOKE signal as the relative change in the intensity $I$ of the transmitted light when the magnetization $\mathbf{M}$ is reversed:

$$
\delta=\frac{I(\mathbf{M})-I(-\mathbf{M})}{I(0)}
$$

where $I(0)=\frac{I(\mathbf{M})+I(-\mathbf{M})}{2}$. We achieve, simultaneously, a large TMOKE signal of $\delta=1.5 \%$ and a high transmittance of $45 \%$ in our experiment. The experimental results 
are supported by numerical simulations. Based on the simulation results, coupling between the localized plasmonic resonance and the photonic waveguide mode is analyzed.

\section{EXPERIMENTAL DEMONSTRATION OF THE ENHANCED TMOKE}

We consider a periodic system of gold nanowires fabricated on a thin film of bismuth iron garnet (BIG), as shown in Fig. 1(a). The 150-nm-thick BIG films are prepared by pulsed-laser deposition [37-39]. Gold nanowires
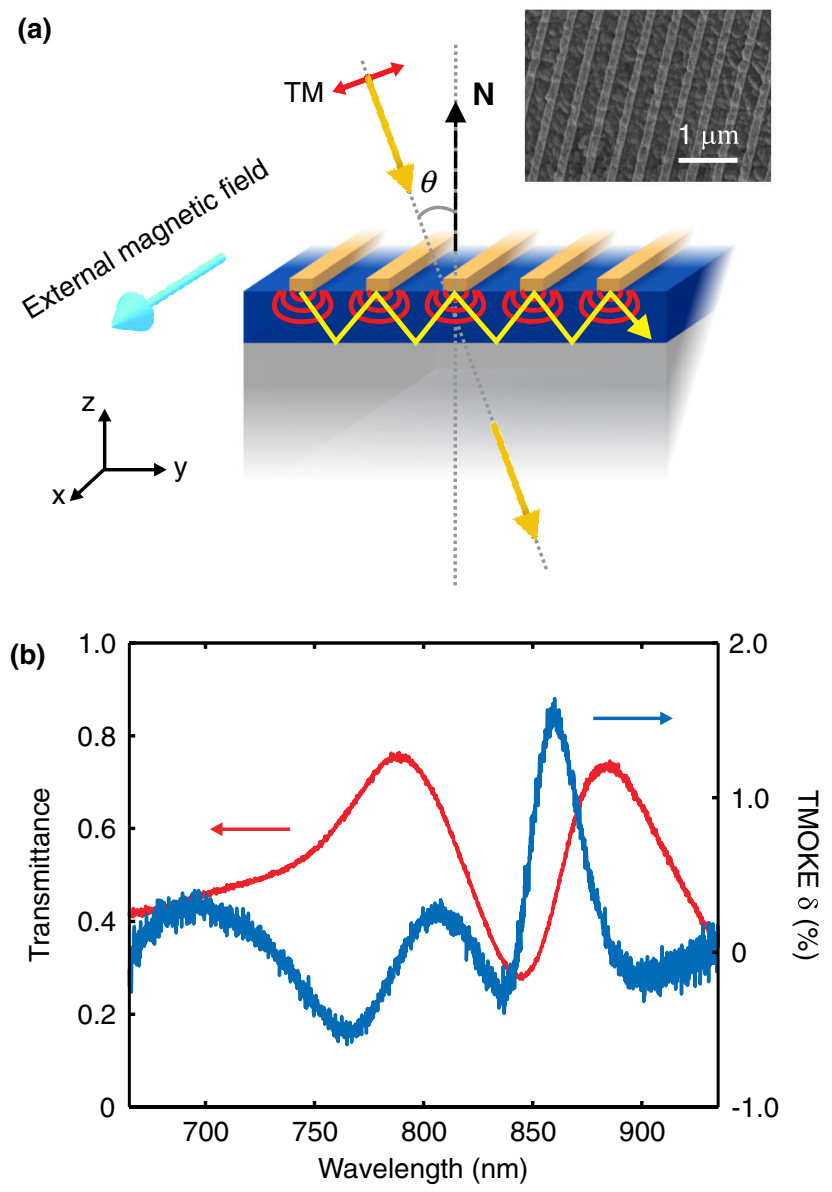

FIG. 1. (a) Schematic of the plasmonic system employed to enhance the transverse magneto-optical Kerr effect. The bismuth iron garnet film (blue) is deposited on glass substrate (grey), and the gold nanowires are structured on top. An external magnetic field $B$ is applied in plane. Obliquely incident light (incident angle $\theta$ ) is polarized perpendicular to the nanowires (TM polarization). Inside the bismuth iron garnet film, the red lines close to the nanowires illustrate the near-field enhancement due to the plasmonic resonance, and the yellow lines illustrate light bouncing up and down inside the waveguide due to the waveguide mode in a simplified geometrical optics analogy. $\mathbf{N}$ is the surface normal of the substrate. The inset shows a scanning electron micrograph of a sample. (b) Measured spectrum of transmittance at a zero $B$ field (red line) and the measured spectrum of the TMOKE signal $\delta$ at $\pm 160 \mathrm{mT}$ (blue line), with a $9^{\circ}$ incident angle for the sample with a 500 -nm period. are structured on the BIG film by electron-beam lithography. Three samples are fabricated with constant width and thickness of the wires but different periodicities. The wire width is $120 \mathrm{~nm}$, the thickness is $65 \mathrm{~nm}$, and the periods are $450 \mathrm{~nm}, 475 \mathrm{~nm}$, and $500 \mathrm{~nm}$, respectively.

The samples are positioned between the ferrite cores of an electromagnet, with the external magnetic field $B$ oriented along the nanowires and the BIG film magnetized in plane, as depicted in Fig. 1(a). A tungsten halogen lamp illuminates the sample at an incident angle $\theta$. The incident light is TM polarized; i.e., its electric field lies in the incidence plane. Light is focused onto the sample with an achromatic doublet, in combination with an adjustable iris to reduce the numerical aperture of the incoming light to less than $1^{\circ}$. The transmitted light is collimated by an achromatic lens and focused on a spectrograph providing a spectral resolution of $0.3 \mathrm{~nm}$.

Figure 1(b) presents the measured transmittance and the measured TMOKE signal for the sample of a 500-nm period. The transmittance spectrum of the structure shows a pronounced resonance at around $850 \mathrm{~nm}$, which only exists for oblique and TM-polarized incident light. The applied magnetic field $B$ along the $+x$ or $-x$ direction modifies the resonance by shifting the resonant wavelength and, at the same time, varying the resonant spectral shape. As a result, a pronounced TMOKE signal is produced [Fig. 1(b)]. It is characterized by the parameter $\delta$ defined by Eq. (1). $\delta$ reaches $1.5 \%$ at $860 \mathrm{~nm}$, where the sample shows rather high transmittance of about $45 \%$. This result constitutes a remarkable improvement with respect to the TMOKE enhanced by SPPs in [3], where the same level of the TMOKE was accompanied by a small transmittance of only $3 \%$. The high transparency is due to the geometrical design of our plasmonic system.

\section{FEATURES OF THE WAVEGUIDE-PLASMON- POLARITON ENHANCED TMOKE}

We first investigate the dependence of the TMOKE on the external magnetic field. By changing the applied magnetic field, we measure the TMOKE signal for five different magnetic field values [Fig. 2(a)]. A progressive increase of the TMOKE signal with the increasing magnetic field can be observed. Extracting the maxima of the TMOKE signals for 24 different values of $B$ [Fig. 2(b)], we further observe that, for $B<10 \mathrm{mT}$, the TMOKE signal changes almost linearly with $B$; when $B$ becomes larger, the trend of the increase starts to saturate. The TMOKE signal reaches the maximum value of $1.5 \%$ at $B=160 \mathrm{mT}$. This is because the TMOKE signal scales with the in-plane magnetization of the film. The magnetization of the BIG gradually starts to saturate when the magnetic field increases over $10 \mathrm{mT}$, and it fully saturates when a magnetic field over $160 \mathrm{mT}$ is applied.

We then change the incident angle $\theta$ from $-9^{\circ}$ to $11^{\circ}$, with a $2^{\circ}$ step, while keeping an external magnetic field of 

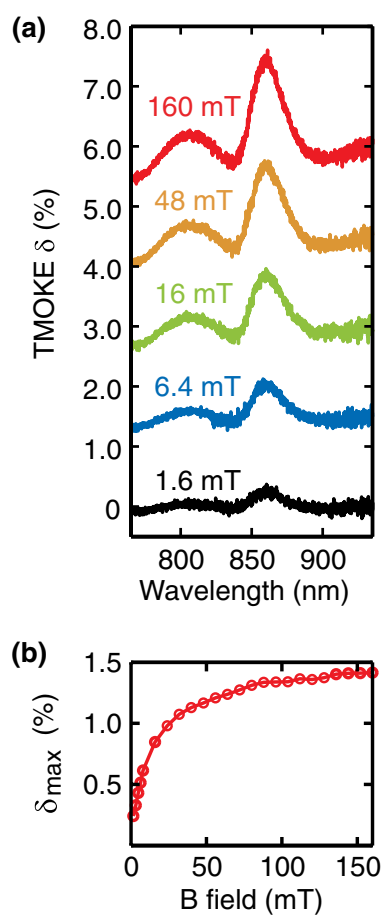

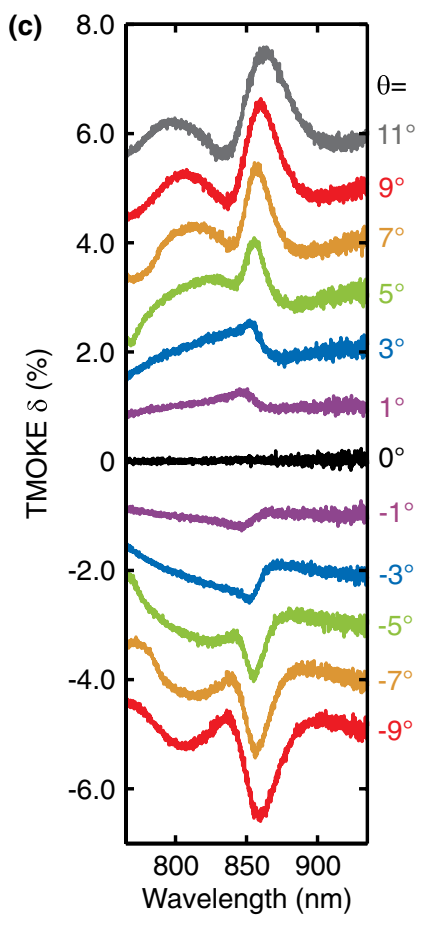

FIG. 2. (a) Measured spectra of the TMOKE signal $\delta$ at different static magnetic fields of $1.6 \mathrm{mT}, 6.4 \mathrm{mT}, 16 \mathrm{mT}, 48 \mathrm{mT}$, and $160 \mathrm{mT}$, respectively. (b) Measured maximum TMOKE signal $\delta_{\max }$ increasing with the external magnetic field. The incident angle is $9^{\circ}$. (c) Measured $\delta$ at different incident angles at a magnetic field of $160 \mathrm{mT}$. From the bottom to the top, the incident angles are $-9^{\circ},-7^{\circ},-5^{\circ},-3^{\circ},-1^{\circ}, 0^{\circ},+1^{\circ}$, $+3^{\circ},+5^{\circ},+7^{\circ},+9^{\circ}$, and $+11^{\circ}$, respectively. The spectra in (a) and (c) are shifted vertically for better visibility.

$160 \mathrm{mT}$, and we measure the TMOKE signal for each angle. The measurement results are shown in Fig. 2(c). One interesting feature of the TMOKE is the odd angular symmetry of $\delta$, which is observed in our experiment. For each pair of incident angles with opposite signs, i.e., $+\theta$ and $-\theta$, the spectra of the TMOKE signals have opposite signs. For normal incidence $\left(\theta=0^{\circ}\right)$, the TMOKE signal vanishes. Increasing the absolute value of the incident angle leads to a rising TMOKE signal, and at incident angles $|\theta|>7^{\circ}$, a saturation of this increase takes place. The spectral position of the resonant feature of the TMOKE signal appears to be insensitive to the incident angle, which is related to the origin of the enhancement of the TMOKE.

While the incident angle has little influence on the resonant wavelength of the TMOKE signal, the period of the nanowires changes the resonant wavelength decisively. We measure the transmittance and the TMOKE signal of the three samples with decreasing periods, as shown in Figs. 3(a) and 3(c). Reducing the grating period shifts the resonant features of both the transmittance and the TMOKE signal to shorter wavelengths, with a gradual decrease of the maximum TMOKE signal $\delta_{\max }$.
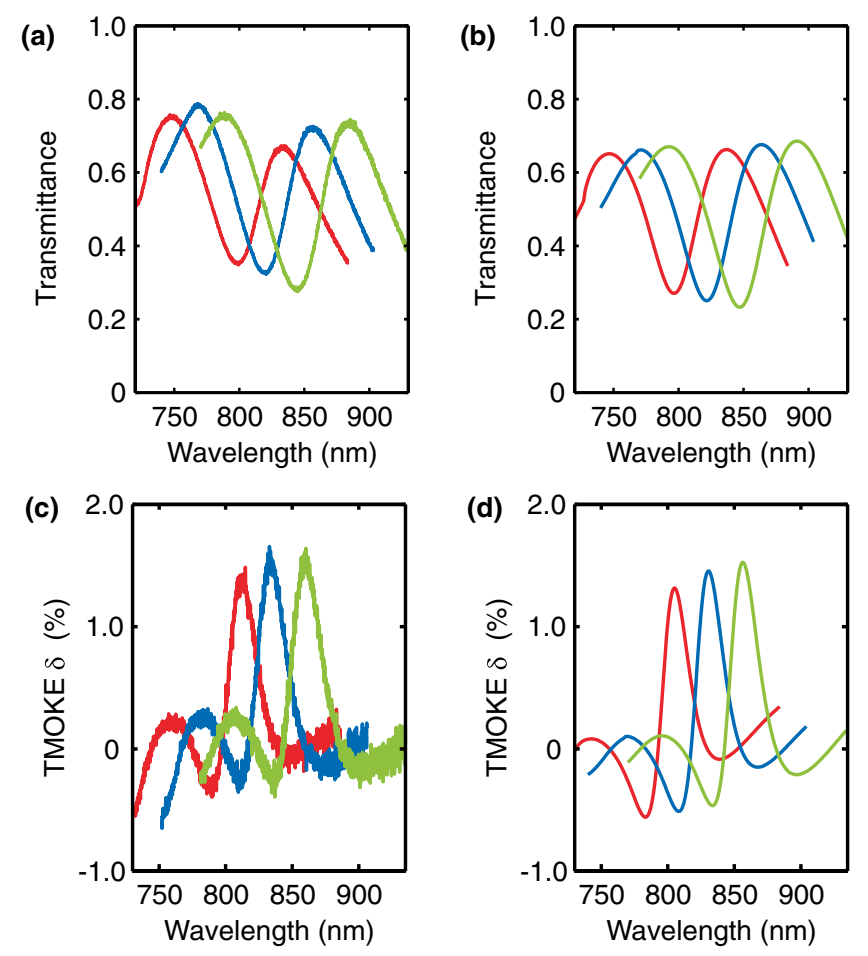

FIG. 3. (a) Measured and (b) simulated transmittance for the structures of a 450-nm period (red lines), a 475-nm period (blue lines), and a 500-nm period (green line). (c) Measured and (d) simulated TMOKE for the structures of a 450-nm period (red lines), a 475-nm period (blue lines), and a 500-nm period (green lines). Illumination is TM polarized with a $9^{\circ}$ incident angle. An external magnetic field of $160 \mathrm{mT}$ is applied.

The transmittance and the TMOKE signal are simulated on the basis of a rigorous coupled wave analysis (RCWA) [40]. The magnetic layer of the structure is described by a dielectric permittivity tensor $\hat{\varepsilon}$ given by $\varepsilon_{i j}=\varepsilon_{i j}^{0}-i e_{i j k} g_{k}$, where $e_{i j k}$ is the Levi-Civita tensor, and $i, j, k=x, y, z$ [2]. $g_{k}$ is related to the magnetization of the BIG film. The material parameters of the BIG used in the RCWA simulations are $\varepsilon_{x x}=\varepsilon_{y y}=$ $\varepsilon_{z z}=6.76+i 0.3, g_{y}=g_{z}=0$, and $g_{x}=0.016-i 0.008$. These parameters are obtained from experimental characterization of the BIG films, and they are averaged over the spectrum from $650 \mathrm{~nm}$ to $950 \mathrm{~nm}$. The dispersion of gold is taken into account [41], and the glass substrate is modeled with a constant permittivity of $\varepsilon=2.13$. In order to model the roughness of the polycrystalline BIG film, which can be observed in the inset of Fig. 1(a), the imaginary parts of the material parameters, $\varepsilon_{i j}^{0}$ and $g_{k}$, are increased by a factor of 5 ; the imaginary part of the gold permittivity in Ref. [41] is increased by a factor of 3 . Numerical simulations are in good agreement with the measurement [Figs. 3(b) and 3(d)].

\section{ANALYSIS OF THE ENHANCEMENT}

In order to understand the enhancement of TMOKE, we take a closer look into the resonant behavior of the hybrid 
structure. On the one hand, TM-polarized light drives a collective oscillation of electrons across each nanowire and excites a particle-plasmon resonance, which enhances the near field. On the other hand, the periodic arrangement of nanowires scatters light into the magneto-optical waveguide layer and excites a TM quasiguided mode [see the illustration in Fig. 1(a)]. When the particle-plasmon resonance and the waveguide mode couple strongly with each other, waveguide-plasmon polaritons are excited $[35,36]$, which enhance the magneto-optical response. Our structures exhibit both a particle-plasmon resonance and a fundamental TM waveguide mode in the spectral range from $700 \mathrm{~nm}$ to $950 \mathrm{~nm}$, where the magneto-optical quality factor of BIG is high.

Assisted by the RCWA simulations, we analyze the eigenmodes of the waveguide-plasmon polaritons. Since the excitation of an eigenmode increases the optical absorbance, the modes can be found as the maxima in the contour diagram of absorbance versus angular frequency $\omega$ and wave number $k$ [Fig. 4(a)]. The diagram of the absorbance reveals one mode with high absorbance at around $\omega=2.2 \times 10^{15} \mathrm{~s}^{-1} \quad(\lambda=860 \mathrm{~nm})$, denoted by LP in Fig. 4(a). It corresponds to the particle-plasmon resonance and is thus dispersionless. The other mode feature, denoted by WG in Fig. 4(a), is a dispersive line, which corresponds to the fundamental TM waveguide mode. When $k$ increases to $k>0.17 \pi / d$, which corresponds to incident angles of $\theta>9^{\circ}$, the two resonances move spectrally closer and start to couple to each other. As the plasmon resonance is broad, the modes already start to couple quite strongly at $\theta=9^{\circ}$.

We simulated the electromagnetic near field and show in Fig. 4(b) the amplitude of the $x$ component of the oscillating magnetic field within one period of the plasmonic waveguide for the sample of a $500-\mathrm{nm}$ period at $9^{\circ}$ incident angle at the maximum TMOKE signal. The two big bright spots inside the magneto-optical waveguide layer are characteristic of the waveguide mode, and the small bright spot at the interface between the gold wire and the waveguide layer is due to the particle plasmons. The near-field distribution is evidence of the strong coupling between the localized particle-plasmon mode and the waveguide mode. The coupling yields the waveguideplasmon polaritons and causes the enhancement of the TMOKE signal when the two resonances are closer to each other [Fig. 4(c)].

Both the waveguide mode and the particle-plasmon resonance are modified by the external magnetic field, but in different ways. For the planar waveguide, we define a normal vector $\mathbf{N}$ to the BIG-glass interface, which is directed along the positive direction of the $z$ axis. In the transversal magnetic configuration, the cross product $\boldsymbol{\tau}=[\mathbf{M} \times \mathbf{N}]$ plays an important role [3,4]. Namely, the effect of nonreciprocity with respect to $\boldsymbol{\tau}$ appears for propagating waveguide modes. The wave number of the waveguide mode is

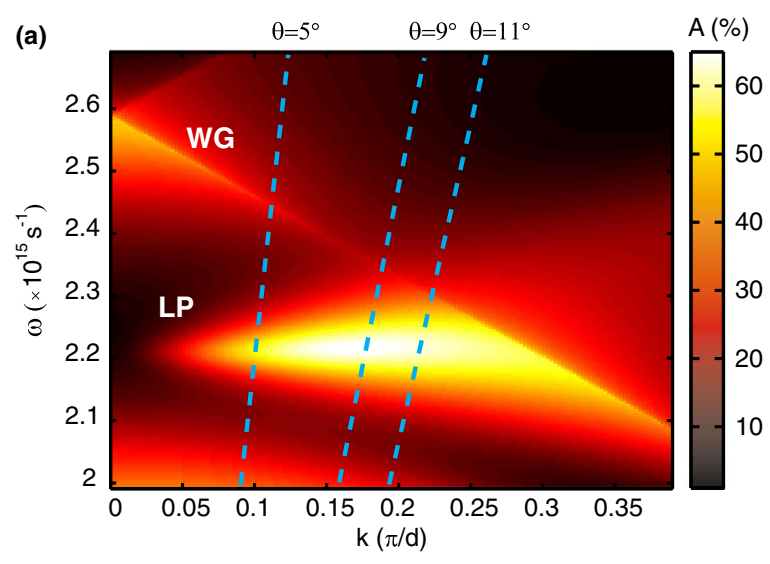

(b)
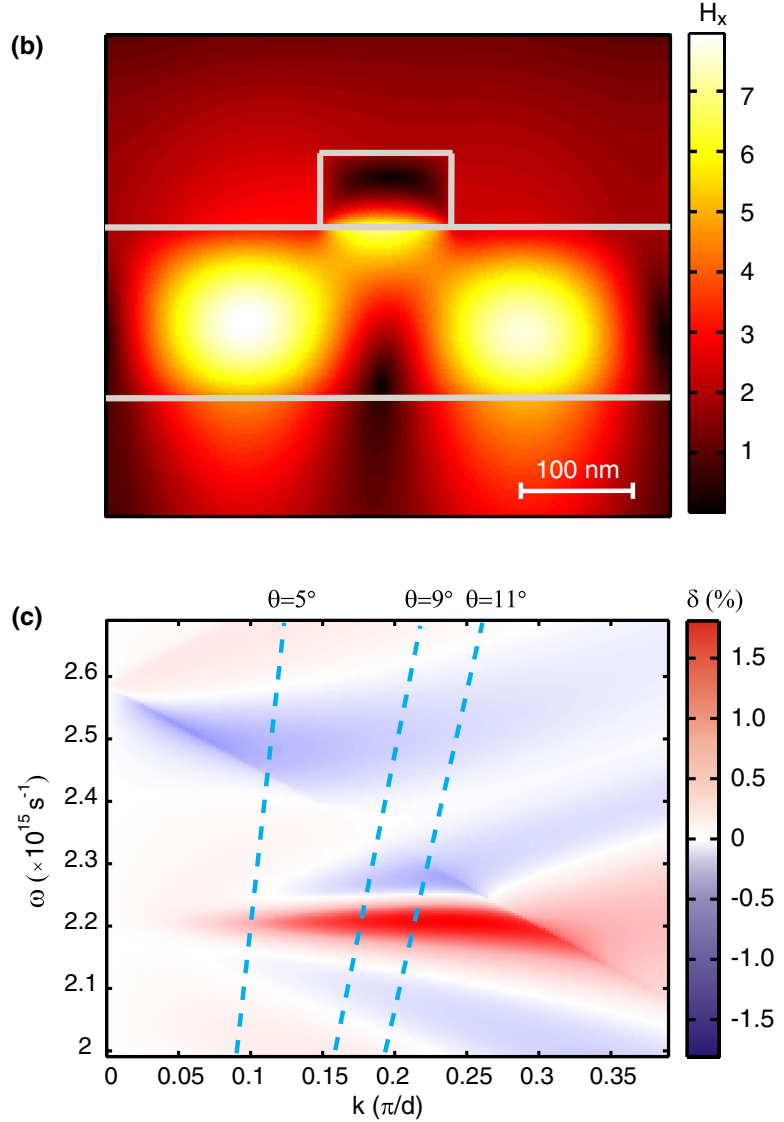

FIG. 4. (a) Simulated dispersion diagram of absorbance $A$ for the sample with a 500-nm period for TM polarization. The blue lines indicate the $\omega-k$ relations at $5^{\circ}, 9^{\circ}$, and $11^{\circ}$ incident angles, respectively. The high absorbance (bright area, denoted by LP) at around $\omega=2.2 \times 10^{15} \mathrm{~s}^{-1}$ is due to the localized plasmon resonance. The dispersive feature in the diagram denoted by WG is due to the waveguide mode. (b) Simulated distribution of the oscillating magnetic field of light $H_{x}$ (amplitude of the $x$ component) along the gold nanowires for the structure of a $500-\mathrm{nm}$ period at a $9^{\circ}$ incident angle and a 860 -nm wavelength $\left(\omega=2.2 \times 10^{15} \mathrm{~s}^{-1}\right)$. The magnetic field is normalized to the amplitude of the incident light magnetic field. The waveguide and the gold nanowire are outlined by grey lines. (c) Simulated $\omega-k$ diagram of the TMOKE signal $\delta$ for the structure of a 500-nm period. The external magnetic field is $160 \mathrm{mT}$, and illumination is TM polarized. 


$$
k=k^{(0)}+\alpha\left(\boldsymbol{\tau} \cdot \mathbf{k}^{(0)}\right)
$$

where $k^{(0)}$ and $\mathbf{k}^{(0)}$ are the wave number and wave vector of the mode when the plasmonic waveguide is not magnetized [42]. Equation (2) dictates that, for a given direction of mode propagation, the change of sign of $\mathbf{M}$ leads to a change of $k$. Because of the mode dispersion $\omega(k)$, this further leads to the change of the eigenmode frequency. Consequently, a transversal magnetic field shifts the resonant features observed in transmission due to the excitation of these modes. This shift gives rise to an S-shaped resonance of the TMOKE signal, which is the differential of the transmittance.

In the case of the localized plasmon resonance, the particle plasmons can be viewed as being formed by standing waves that are the sum of two waves propagating in opposite directions. As the contributions of magnetization for the two counterpropagating waves average to zero, the eigenmode frequencies of the localized modes are hardly influenced by the magnetization. However, the magnetization modifies the nondiagonal terms in the permittivity tensor of the magnetic film and thus changes the boundary conditions at the two interfaces of the magnetic film. This modification mainly leads to a change of the maximum and minimum values in the transmission and reflection spectra. As a result, the TMOKE signal caused by localized particle plasmons exhibits Lorentzian-like spectra.

The prominent feature of the plasmonic waveguide is that the particle-plasmon resonance and the waveguide mode are excited simultaneously. In this case, the magnetic field induces both a shift and a deformation to the resonant spectrum of transmittance. The presence of the two mechanisms contributing to the TMOKE explains the observed enhancement when the two modes hybridize. The spectral feature of $\delta$ is a combination of the S- and Lorentzian-like line shapes, as seen in Figs. 1, 2(a), 2(c), and 3(b).

\section{CONCLUSIONS}

We have demonstrated a novel way to induce large TMOKE by using a sophisticated structure that incorporates waveguide-plasmon polaritons with a magnetooptical material. The waveguide-plasmon polaritons are formed by coupling particle plasmons with a planar photonic waveguide. Flipping the magnetic field changes the magnetization and thus causes changes to both the particle-plasmon resonance and the waveguide modes. When resonances of the localized plasmons and the propagating waveguide modes hybridize, the TMOKE signal reaches $1.5 \%$. This signal is, so far, the highest TMOKE in combination with high transparency that has been experimentally demonstrated. Further simulation predicts that improving the quality of the magneto-optical thin film can increase the TMOKE signal of our design to $10 \%$.

Additionally, waveguide-plasmon polaritons provide us with high degrees of freedom to engineer the dispersion by geometrical parameters, which is hard to achieve otherwise. As a result, we can engineer the working wavelength conveniently by tuning the period, as demonstrated in both experiment and simulation (Fig. 3).

Using the interconnection between the TMOKE and the magnetization of the film, plasmonically enhanced TMOKE can be applied to probe the magnetization states of thin films whose innate magneto-optical effects are too small for convenient measurement. Our concept also has potential applications in thin-film optical devices of magneto-optical switching and modulation, which could be used in future photonic circuits or fiber opticalcommunication systems.

\section{ACKNOWLEDGMENTS}

This work was supported by DFG (Grants No. SPP1391, No. FOR730, No. GI 269/11-1, and No. AK40/7-1), BMBF (Grant No. 13N10146, and FARADAY, Grant No. FKZ 13N12443), ERC (Advanced Grant COMPLEXPLAS), MWK, Baden-Württemberg Stiftung, RFBR (Projects No. 12-02-33100, No. 13-02-01122, No. 13-02-91334, No. 13-02-90438, and No. 13-02-92710), and the Russian Federal Targeted Program "Scientific and ScientificPedagogical Personnel of the Innovative Russia." This work was also supported by the German Research Foundation (DFG) within the funding program Open Access Publishing. J. Y. C. and D. D. acknowledge support by the Carl-Zeiss-Stiftung. V. I. B. acknowledges support by the Alexander von Humboldt Foundation. We gratefully thank Sven Hein for his support.

[1] J. P. Liu, E. Fullerton, O. Gutfleisch, and D. J. Sellmyer, Nanoscale Magnetic Materials and Applications (Springer, Dordrecht-Heidelberg-London-New York, 2009).

[2] A. Zvezdin and V. Kotov, Modern Magnetooptics and Magnetooptical Materials (Tay \& Francis, New YorkOxen, 1997).

[3] V. I. Belotelov, I. A. Akimov, M. Pohl, V. A. Kotov, S. Kasture, A.S. Vengurlekar, Achanta Venu Gopal, D. R. Yakovlev, A.K. Zvezdin, and M. Bayer, Enhanced Magneto-Optical Effects in Magnetoplasmonic Crystals, Nat. Nanotechnol. 6, 370 (2011).

[4] M. Pohl, L.E. Kreilkamp, V. I. Belotelov, I. A. Akimov, A. N. Kalish, N.E. Khokhlov, V.J. Yallapragada, A. V. Gopal, M. Nur-E-Alam, M. Vasiliev, D. R. Yakovlev, K. Alameh, A. K. Zvezdin, and M. Bayer, Tuning of the Transverse Magneto-Optical Kerr Effect in MagnetoPlasmonic Crystals, New J. Phys. 15, 075024 (2013).

[5] V. I. Belotelov, D. A. Bykov, L. L. Doskolovich, A. N. Kalish, and A.K. Zvezdin, Giant Transversal Kerr Effect in Magneto-Plasmonic Heterostructures: The Scattering Matrix Method, J. Exp. Theor. Phys. 110, 816 (2010).

[6] J. B. González-Díaz, B. Sepúlveda, A. García-Martín, and G. Armelles, Cobalt Dependence of the Magneto-Optical 
Response in Magnetoplasmonic Nanodisks, Appl. Phys. Lett. 97, 043114 (2010).

[7] V. I. Belotelov, L.L. Doskolovich, and A. K. Zvezdin, Extraordinary Magneto-Optical Effects and Transmission through Metal-Dielectric Plasmonic Systems, Phys. Rev. Lett. 98, 077401 (2007).

[8] J.C. Banthí, D. Meneses-Rodríguez, F. García, M. U. González, A. García-Martín, A. Cebollada, and G. Armelles, High Magneto-Optical Activity and Low Optical Losses in Metal-Dielectric Au/Co/Au$\mathrm{SiO}_{2}$ Magnetoplasmonic Nanodisks, Adv. Mater. 24, OP36 (2012).

[9] V. V. Temnov, G. Armelles, U. Woggon, D. Guzatov, A. Cebollada, A. Garcia-Martin, J. Garcia-Martin, T. Thomay, A. Leitenstorfer, and R. Bratschitsch, Active Magneto-Plasmonics in Hybrid Metal-Ferromagnet Structures, Nat. Photonics 4, 107 (2010).

[10] V. V. Temnov, Ultrafast Acousto-Magneto-Plasmonics, Nat. Photonics 6, 728 (2012).

[11] K. Fang, Z. Yu, V. Liu, and S. Fan, Ultracompact Nonreciprocal Optical Isolator Based on Guided Resonance in a Magneto-Optical Photonic Crystal Slab, Opt. Lett. 36, 4254 (2011).

[12] L. Bi, J. Hu, P. Jiang, D. H. Kim, G. F. Dionne, L. C. Kimerling, and C. A. Ross, On-Chip Optical Isolation in Monolithically Integrated Non-Reciprocal Optical Resonators, Nat. Photonics 5, 758 (2011).

[13] A. R. Davoyan, A. M. Mahmoud, and N. Engheta, Optical Isolation with Epsilon-Near-Zero Metamaterials, Opt. Express 21, 3279 (2013).

[14] J. Y. Chin, T. Steinle, T. Wehlus, D. Dregely, T. Weiss, V. I. Belotelov, B. Stritzker, and H. Giessen, Nonreciprocal Plasmonics Enables Giant Enhancement of Thin-Film Faraday Rotation, Nat. Commun. 4, 1599 (2013).

[15] A. R. Davoyan and N. Engheta, Nonreciprocal Rotating Power Flow within Plasmonic Nanostructures, Phys. Rev. Lett. 111, 047401 (2013).

[16] A. Davoyan and N. Engheta, Nanoscale Plasmonic Circulator, New J. Phys. 15, 083054 (2013).

[17] A. B. Khanikaev, S.H. Mousavi, G. Shvets, and Y.S. Kivshar, One-Way Extraordinary Optical Transmission and Nonreciprocal Spoof Plasmons, Phys. Rev. Lett. 105, 126804 (2010).

[18] V. Bonanni, S. Bonetti, T. Pakizeh, Z. Pirzadeh, J. Chen, J. Nogués, P. Vavassori, R. Hillenbrand, J. Åkerman, and A. Dmitriev, Designer Magnetoplasmonics with Nickel Nanoferromagnets, Nano Lett. 11, 5333 (2011).

[19] J. Chen, P. Albella, Z. Pirzadeh, P. Alonso-González, F. Huth, S. Bonetti, V. Bonanni, J. Åkerman, J. Nogués, P. Vavassori, A. Dmitriev, J. Aizpurua, and R. Hillenbrand, Plasmonic Nickel Nanoantennas, Small 7, 2341 (2011).

[20] N. Maccaferri, J. B. González-Díaz, S. Bonetti, A. Berger, M. Kataja, S. van Dijken, J. Nogués, V. Bonanni, Z. Pirzadeh, A. Dmitriev, J. Åkerman, and P. Vavassori, Polarizability and Magnetoplasmonic Properties of Magnetic General Nanoellipsoids, Opt. Express 21, 9875 (2013).

[21] V. I. Belotelov, L. E. Kreilkamp, I. A. Akimov, A. N. Kalish, D. A. Bykov, S. Kasture, V. J. Yallapragada, Achanta Venu Gopal, A. M. Grishin, S. I. Khartsev, M. Nur-E-Alam, M. Vasiliev, L. L. Doskolovich,
D. R. Yakovlev, K. Alameh, A. K. Zvezdin, and M. Bayer, Plasmon-Mediated Magneto-Optical Transparency, Nat. Commun. 4, 2128 (2013).

[22] A. A. Grunin, A. G. Zhdanov, A.A. Ezhov, E. A. Ganshina, and A. A. Fedyanin, Surface-Plasmon-Induced Enhancement of Magneto-Optical Kerr Effect in AllNickel Subwavelength Nanogratings, Appl. Phys. Lett. 97, 261908 (2010).

[23] M. V. Sapozhnikov, S. A. Gusev, B. B. Troitskii, and L. V. Khokhlova, Optical and Magneto-Optical Resonances in Nanocorrugated Ferromagnetic Films, Opt. Lett. 36, 4197 (2011).

[24] G. Ctistis, E. Papaioannou, P. Patoka, J. Gutek, P. Fumagalli, and M. Giersig, Optical and Magnetic Properties of Hexagonal Arrays of Subwavelength Holes in Optically Thin Cobalt Films, Nano Lett. 9, 1 (2009).

[25] D. M. Newman, M. L. Wears, R. J. Matelon, and I. R. Hooper, Magneto-Optic Behaviour in the Presence of Surface Plasmons, J. Phys. Condens. Matter 20, 345230 (2008).

[26] J. B. González-Díaz, A. García-Martín, G. Armelles, J. M. García-Martín, C. Clavero, A. Cebollada, R. A. Lukaszew, J.R. Skuza, D. P. Kumah, and R. Clarke, SurfaceMagnetoplasmon Nonreciprocity Effects in Noble-Metal/ Ferromagnetic Heterostructures, Phys. Rev. B 76, 153402 (2007).

[27] G. Armelles, A. Cebollada, A. García-Martín, J. M. García-Martín, M. U. González, J. B. González-Díaz, E. Ferreiro-Vila, and J.F. Torrado, Magnetoplasmonic Nanostructures: Systems Supporting Both Plasmonic and Magnetic Properties, J. Opt. A 11, 114023 (2009).

[28] M. G. Manera, G. Montagna, E. Ferreiro-Vila, L. GonzálezGarcía, J. R. Sánchez-Valencia, A. R. González-Elipe, A. Cebollada, J. M. Garcia-Martin, A. Garcia-Martin, G. Armelles, and R. Rella, Enhanced Gas Sensing Performance of $\mathrm{TiO}_{2}$ Functionalized Magneto-Optical SPR Sensors, J. Mater. Chem. 21, 16049 (2011).

[29] C. Clavero, K. Yang, J. R. Skuza, and R. A. Lukaszew, Magnetic-Field Modulation of Surface Plasmon Polaritons on Gratings, Opt. Lett. 35, 1557 (2010).

[30] G. Armelles, J.B. González-Díaz, A. García-Martín, J. M. García-Martín, A. Cebollada, M. U. González, S. Acimovic, J. Cesario, R. Quidant, and G. Badenes, Localized Surface Plasmon Resonance Effects on the Magneto-Optical Activity of Continuous Au/Co/Au Trilayers, Opt. Express 16, 16104 (2008).

[31] J. F. Torrado, J. B. González-Díaz, G. Armelles, A. GarcíaMartín, A. Altube, M. López-García, J. F. Galisteo-López, A. Blanco, and C. López, Tunable Magneto-Photonic Response of Nickel Nanostructures, Appl. Phys. Lett. 99, 193109 (2011).

[32] R. K. Hickernell and D. Sarid, Long-Range Surface Magnetoplasmons in Thin Nickel Films, Opt. Lett. 12, 570 (1987).

[33] G.C. Aers and A.D. Boardman, The Theory of Semiconductor Magnetoplasmon-Polariton Surface Modes: Voigt Geometry, J. Phys. C 11, 945 (1978).

[34] M.S. Kushwaha, Plasmons and Magnetoplasmons in Semiconductor Heterostructures, Surf. Sci. Rep. 41, 1 (2001).

[35] A. Christ, S. G. Tikhodeev, N. A. Gippius, J. Kuhl, and H. Giessen, Waveguide-Plasmon Polaritons: Strong Coupling 
of Photonic and Electronic Resonances in a Metallic Photonic Crystal Slab, Phys. Rev. Lett. 91, 183901 (2003).

[36] A. Christ, T. Zentgraf, J. Kuhl, S. G. Tikhodeev, N. A. Gippius, and H. Giessen, Optical Properties of Planar Metallic Photonic Crystal Structures: Experiment and Theory, Phys. Rev. B 70, 125113 (2004).

[37] R. Lux, A. Heinrich, S. Leitenmeier, T. Körner, M. Herbort, and B. Stritzker, Pulsed-Laser Deposition and Growth Studies of $\mathrm{Bi}_{3} \mathrm{Fe}_{5} \mathrm{O}_{12}$ Thin Films, J. Appl. Phys. 100, 113511 (2006).

[38] T. Wehlus, T. Körner, S. Leitenmeier, A. Heinrich, and B. Stritzker, Magneto-Optical Garnets for Integrated Optoelectronic Devices, Phys. Status Solidi (a) 208, 252 (2011).
[39] J. Griesbauer, T. Körner, T. Wehlus, A. Heinrich, B. Stritzker, J. Simon, and W. Mader, Mechano- and Magneto-Optical Sensitivity of YIG Buffer Systems, CrystEngComm 13, 77 (2011).

[40] L. Li, Fourier Modal Method for Crossed Anisotropic Gratings with Arbitrary Permittivity and Permeability Tensors, J. Opt. A 5, 345 (2003).

[41] P. B. Johnson and R. W. Christy, Optical Constants of the Noble Metals, Phys. Rev. B 6, 4370 (1972).

[42] V. I. Belotelov, I. A. Akimov, M. Pohl, A. N. Kalish, S. Kasture, A. S. Vengurlekar, A. V. Gopal, V. A. Kotov, D. Yakovlev, A. K. Zvezdin, and M. Bayer, Intensity Magnetooptical Effect in Magnetoplasmonic Crystals, J. Phys. Conf. Ser. 303, 012038 (2011). 